University of Nebraska - Lincoln

DigitalCommons@University of Nebraska - Lincoln

20th \& 21st Century French and Francophone Modern Languages and Literatures, Department Studies International Colloquium

3-16-2020

\title{
« Ceindre la Terre »: sur quelques tours du monde
}

Jean-François Duclos

Metropolitan State University of Denver, jduclos@msudenver.edu

Follow this and additional works at: https://digitalcommons.unl.edu/ffsc2020

Part of the Comparative Literature Commons, French and Francophone Literature Commons, and the Other French and Francophone Language and Literature Commons

Duclos, Jean-François, "« Ceindre la Terre » : sur quelques tours du monde" (2020). 20th \& 21st Century French and Francophone Studies International Colloquium. 3.

https://digitalcommons.unl.edu/ffsc2020/3

This Article is brought to you for free and open access by the Modern Languages and Literatures, Department of at DigitalCommons@University of Nebraska - Lincoln. It has been accepted for inclusion in 20th \& 21st Century

French and Francophone Studies International Colloquium by an authorized administrator of DigitalCommons@University of Nebraska - Lincoln. 
20th \& 21st Century French \& Francophone Studies Colloquium University of Nebraska-Lincoln, March 2020

Session 9. B - Le voyage

\author{
«Ceindre la Terre » : sur quelques tours du monde \\ Jean-François Duclos \\ Metropolitan State University of Denver
}

« La vie est ronde », écrit Joë Bousquet en 1946. Le constat, cela va sans dire, est le même s'agissant de la Terre et nourrit l'imaginaire depuis qu'on tient le fait pour acquis. « Métaphore du cercle » de Robert Poulet, "phénoménologie du rond » de Gaston Bachelard, et depuis quelque temps l'idée de «planétarité » : chaque époque offre le moyen de spéculer sur ce que, dans leur essai intitulé «The Planetary Condition», Elias et Moraru décrivent comme «a structure of awareness ${ }^{1} »$. Se soumettre à l'évidence de cette géométrie constitue une manière d'être qui est aussi une façon de sentir et de penser, de se penser dans l'espace de nos existences. Les remarques qui suivent ont pour but d'examiner la manière dont quatre écrivains français d'aujourd'hui (Olivier Rolin, Jacques Réda, Tanguy Viel et Christian Garcin) envisagent consciemment le monde comme planète. Plus précisément, je souhaite m'interroger sur leurs textes qui, prenant le monde comme espace physique et textuel, proposent d'en faire le tour.

Dans L'invention du monde², Olivier Rolin organise mille récits d'un même jour sur Terre, le 21 mars 1989. Tous les faits qui y sont relatés sont tirés de la consultation par l'auteur de

\footnotetext{
${ }^{1}$ Amy J. Elias et Christian Moraru. "Introduction: The Planetary Condition", in Elias and Moraru (ed.), The Planetary Turn. Relationality and Geoesthetics in the Twenty-First Century. Evanston, Illinois: Northwestern University Press, 2015, p. xi.

${ }^{2}$ Olivier Rolin. L'invention du monde (1993) in Circus I. Romans, récits, articles (1980-1998). Paris : Le Seuil, 2011.
} 
centaines de journaux publiés sur tous les continents le lendemain de ce jour d'équinoxe. La matière du monde se lie et se délie sous l'œil panoptique à la fois précis et délirant d'un dieu gouailleur, pétri du désir de saisir et de palper, de séduire et d'aimer. Les crimes, les catastrophes naturelles ou humaines, ces « histoires de grands et beaux sentiments (...) qui cour[ent] les rues de la terre » (1232), ces « millions de récritures simultanées », forment la matière de quarante-huit chapitres, soit le temps qu'il faut pour que toutes les heures d'un seul jour se déclinent sur chaque méridien (1243). «Je vais », écrit-il, « faire un portrait du monde [...]. Le tour du monde en un jour. » (1094). Le réel se fait ainsi lisible et visible, de part en part (1097). En reliant thématiquement plutôt que géographiquement les faits et les histoires, le narrateur s'autorise toutes les virtuosités. Il n'est pas là pour soupeser les âmes ou construire des paysages, mais pour prouver par l'exemple de ses propres contorsions géographiques que « chaque point de l'espace se trouve lié, tressé à tous les autres en lesquels il se transforme, chaque point du temps aussi n'est séparé des autres que par un point de vue, une irisation » (1162). L'expression « et maintenant », maintes fois reprise au cours des cinq cents pages de ce roman sans fiction, dit moins la succession des instants que le fait que tout se passe ou tout est susceptible de passer au même moment sur tous les points du globe. «C'est moi qui suis celui qui voit tout, par qui la terre voit tout, l'œil du monde », s'exclame Apollon, par la bouche d'Ovide, que reprend le narrateur de Rolin.

En 2018, Tanguy Viel et Christian Garcin entreprennent tour du monde « sans avion », tour dont ils font la relation dans un volume écrit à quatre mains intitulé Travelling ${ }^{3}$. Les images et les rencontres que convoquent les deux auteurs sont tout autant tirées d'une expérience vécue durant les trois mois qu'a duré leur voyage pour parcourir trois continents et deux océans que d'une vision déjà abondamment enrichie par une culture livresque et cinématographique. Aussi s'agit-il, avant

\footnotetext{
${ }^{3}$ Christian Garcin et Tanguy Viel. Travelling. Un tour du monde sans avion. Paris : JC Lattès, 2019.
} 
même de quitter son domicile, de mentalement « ceindre la Terre », de faire « le cercle entier du $40^{\mathrm{e}}$ parallèle », pour domestiquer aussi bien son propre parcours à venir que le territoire bientôt parcouru. En décidant de rester à sa surface, pour rouler sur son sol ou fendre ses flots, les voyageurs envisagent leur périple comme un très long travelling, sans à-coup ni zigzag; aux antipodes, si l'on peut dire, du texte de Rolin. Envisager ainsi la Terre, se rapprocher de ceux qui y vivent, c'est d'abord « rêver cette longue phrase », écrit Viel ou Garcin, « la distance se réduisait doucement, domestiquée par la pensée nocturne » (21). «Domicile », « domestiquer » : il s'agit de faire sien le monde et sa représentation, non en le gardant à distance mais en formant un cercle autour de lui. Le mouvement est trop lent, et à dessein, pour faire de « la cage des méridiens ${ }^{4} »$ le lieu d'une course. Surtout convient-il de ne pas aller trop vite, au contraire, afin de prouver qu'il est encore possible de faire le tour du monde en plus de quatre-vingts jours.

Toute la difficulté de l'exercice ne réside pas dans l'idée de traverser des paysages mais dans la recherche du moyen le plus efficace de saisir l'esprit d'un lieu dans sa globalité, et de se saisir soi-même comme être planétaire. Qu'il s'agisse d'une ville, d'une région, ou d'un continent entier, le regard est mis au défi de sa représentation. S'il est question de distance dans ce livre, ce n'est pas tant celle que parcourent Garcin et Viel, à la vitesse du train ou du cargo, mais celle qui séparent l'imaginaire envisagé de chez soi et le réel perçu sur place. Il est moins question de découverte et d'émerveillement que de mise en relation du réel avec sa représentation partagée. Comment penser l'Amérique du point de vue des Amérindiens ? La synecdoque peut-elle devenir le régime d'élection d'une vision du Japon ? Comment trouver une forme de profondeur à la Russie ? Toutes ces questions contribuent à dessiner, « dans l'espace mental de nos géographies

\footnotetext{
${ }^{4}$ Blaise Cendrars, «Le Panama ou les aventures de mes sept oncles [1918] », in Du Monde entier. Poésies complètes 1912-1924. Paris : Gallimard, coll. « Poésie », 1993, p. 46.
} 
intimes une nouvelle géométrie qui, loin d'abolir les différences, les révèle et les rehausse selon une mesure inédite » (244).

À mi-chemin chronologique de ceux deux textes, Jacques Réda, dans un court chapitre de son recueil Les Cinq point cardinaux ${ }^{5}$, met lui aussi en scène un être palpeur et curieux mais qui, au lieu de se lancer sur les routes, se contemple de ramasser en automne un marron d'Inde dans un jardin parisien afin de méditer plus tard sur l'unité du globe. Le dieu, dans ce texte, n'est pas celui qui voyage par la pensée comme chez Rolin, mais celui qui fait un présent à l'adulte afin qu'il accomplisse, je cite « un acte de piété personnelle, qui renouvelle et sacralise un mouvement spontané des enfants » (25). Sorti du tiroir où il l'a laissé toute une saison, le marron donne à Réda l'occasion d'éprouver sous ses doigts et dans sa paume «la ronde unité du monde ». Toute une ambiance liée à la Rome ancienne parcourt ce texte comme pour revenir à un imaginaire scolaire ainsi qu'aux fondamentaux de nos connaissances lucrétiennes sur la nature des choses. À la fois modeste symbole, « objet d'art naturel » (27), le marron est remplaçable, collectionnable, jetable aussi. Mais quel dommage, et peut-être quel sacrifice, car sa « petite sphère cabossée » fait d'abord penser une tête protégeant comme elle le peut un cerveau. La tenir entre ses doigts ou au fond de sa paume, sentir sa «marque d'une fontanelle grisâtre un peu rugueuse » (27), permet, précise Réda, « d'instruire l'âme par le biais de la sensualité. »

Le marron comme cerveau constitue la première étape sensible d'une métamorphose destinée, par l'acte de tenir, de sentir, de manier, de caresser et de voir, à s'élever métaphysiquement. Au cours de ce processus, ce n'est ni l'esprit de l'être qui pense et qui agit, ni la Terre elle-même où il se meut, mais une graine, entité destinée à devenir un arbre, qui à son tour fera tomber ses marrons à l'automne comme s'il en pleuvait. La « ronde unité du monde » (27)

\footnotetext{
${ }^{5}$ Jacques Réda. Les Cinq points cardinaux. Montpellier : Fata Morgana, 2003.
} 
qu'imagine Réda en contemplant un marron n'est pas le monde lui-même mais son fruit. Non pas le fragment d'une totalité mais une totalité déjà contenue, toujours en devenir. Le cinquième point cardinal auquel le titre du volume fait référence est intérieur : c'est un nouvel horizon qui englobe les quatre autres. À la fois observable et hors de portée de la vue, il renvoie à une conscience, une «awareness ». Les hémisphères de la Terre se confondent, mais seulement le temps d'y penser, avec ceux de nos cerveaux.

Dans ces trois textes, la saisie du monde se conçoit comme geste total. Il s'agit de tout voir et tout comprendre dans le même mouvement de pensée géographique, sans distinction d'échelle. Il faut dire qu'en occident, et peut-être pas qu'en occident d'ailleurs ${ }^{6}$, le rêve est ancien, avant de se consolider dans les sciences à travers la probabilité de Laplace. Pour le mathématicien du début du dix-neuvième siècle, toute chose présente, si infime soit-elle, fait partie d'un tout rattaché par «l'effet de son état antérieur et comme cause de ce qui va suivre ${ }^{7}$.» Une intelligence qui connaitrait toutes les forces dont la nature est animée, et qui serait capable de les saisir en un instant, serait alors capable d'embrasser le monde tout entier jusque ses plus lointains atomes. « Rien », dans ce cas, « ne serait incertain », et « l'avenir comme le passé » serait présent aux yeux de celui que Laplace désigne comme le plus prodigieux des esprits.

Pour Rolin et ses avatars panoptiques (en 1989, l'internet n'avait pourtant pas encore eu le temps de se déployer), «le monde n’est que ça, une grosse boule de gaz fictif» crépitante d'informations sur laquelle il convient de brancher son «système nerveux de fibres optique, un flamboyant magasin de miroirs» (1094). Toutes les formes de la totalité sont ainsi

\footnotetext{
${ }^{6}$ Les poètes chinois ne rêvent-ils pas d'écrire la description du monde entier sur un seul grain de riz ?

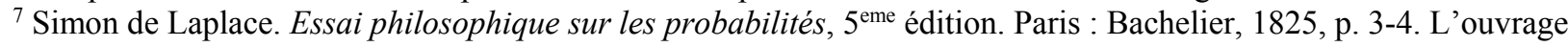
est disponible sur gallica.fr. Pour Laplace, les « questions les plus importantes de la vie » ne sont « en effet, pour la plupart, que des problèmes de probabilité », p. 1.
} 
convoquées pour être décodées : la goutte d'eau où se reflèterait un instant l'image d'un «monde fulgurant de signes, où tout se lisait dans tout, tout s'échangeait dans tout» (1105); l'« aleph autogène » générant constamment ses propres paroles; la « bibliothèque immense » (1112) où se reflètent les images; le « théâtre du monde » où se jouerait tous les drames possibles, etc. « Je ne sais pas si vous vous rendez compte de ce que ça veut dire, TOUT : comment vous en rendriezvous compte ? » demande le narrateur de L'Invention du monde à ses lecteurs.

Dans ce surplomb omniscient, le monde mis sous tension se transforme en une « une sphère bien cousue d'histoires, aux imprévisibles rebonds » (1139). Même si les tragédies se ressemblent, même si les crimes se succèdent selon les mêmes modes opératoires pour les mêmes futiles raisons, même s'il s'agit à chaque rencontre amoureuse de s'illusionner sur son unicité, chaque jour est broyé, découpé en fragments, en paillettes infimes qui, combinées tout à fait autrement, recomposent ailleurs de nouvelles scènes. Nous ne faisons jamais deux fois la même chose, et la force de ce roman hors norme est d'en fournir la preuve sur le mode paradoxal de la répétition.

Le monde, d'abord conçu comme une page où l'on y voit s'inscrire «la baroque calligraphie des villes » (1169) se déploie progressivement comme le lieu même du langage. Un échange s'opère, par lequel l'unité émerge entre « entre un sonnet de Shakespeare et les montagnes Rocheuses ; un mot prononcé en rêvant par un lépreux endormi sur un trottoir de Bombay et les arabesques bleues du Luro Shivo, les pages jaunes de l'annuaire du téléphone et le mouvement des navires sur les mers du globe, les lignes qui filent sous ma plume et la face du monde. » (1189)

Pour Jacques Réda, ce rêve panoptique l'a amené des décennies avant Les cinq point cardiaux à imaginer une équivalence entre le ciel et l'œil, l'univers et le cerveau. La machine qu'il invente pour la circonstance, ou plutôt qu'il s'imagine inventer, ressemble à un immense 
télescope ${ }^{8}$. L'œil collé sur la dernière de ses lunettes y voit tout, ou pour reprendre les propos de Rolin, absolument tout. "Tu mettrais l'univers dans ta ruelle», écrit Baudelaire; tu mettras l'univers dans ta prunelle, a-t-on envie de dire. Mais cette saisie, tout nécessaire soit-elle, relève d'une volonté hégémonique qui enfreint la conscience de l'écrivain. Il doit vite abandonner et s'habituer au fait que le monde se présente par fragments.

Tout rêve de maîtrise prend le risque d'inscrire son épuisement en filigrane. Le prodigieux esprit de Laplace, qui veut se saisir du monde pour l'expliquer, est aussi un démon. «Pour ce démon », écrit Bertrand Westphal dans son essai consacré à la littérature et l'art contemporain face à la globalisation, "l'univers devient perceptible et intelligible dans ses moindres recoins. L'existence est sans surprise pour lui, son savoir si infini qu'il prévoit tous les scénarios ${ }^{9} »$ Si bien qu'aller voir sur place à quoi peut bien ressembler un monde déjà connu de tous, c'est immanquablement prendre le risque de se perdre dans ses propres représentations. Entre désir de tout voir à partir du point fixe de sa chambre (ou du jardin du Luxembourg) et celui d'aller y voir soi-même, un point d'équilibre doit se trouver, qui contient en germe le risque de la mélancolie.

\footnotetext{
${ }^{8}$ Jacques Réda. «Physique de la poésie » in Celle qui vient à pas légers. Montpellier : Fata Morgana, 1985, 1999. Voir Jean-François Duclos. « Tu mettrais l'univers tout entier dans ta prunelle : le ciel et l'œil de Jacques Réda. » Cincinnati Romance Review, numéro 32, 2011.

${ }^{9}$ Bertrand Westphal. La Cage des méridiens. La littérature et l'art contemporain face à la globalisation. Paris: Minuit, 2016, pp. 162-163.
} 